\title{
Predistortion for Solid State Amplifier of Mobile Radio Systems
}

\author{
Henryk Gierszal, Witold Hohubowicz and Przemysław Sulek \\ Institute of Communication and Information Technologies in Poznań, \\ Palacza 91A, 60-273 Poznań, Poland \\ University of Technology and Agriculture in Bydgoszcz \\ Kaliskiego 7, 85-763 Bydgoszcz, Poland
}

Key words: predistortion, nonlinearity, mobile system, amplifier, compensation for nonlinearity, $3^{\text {rd }}$ generation system, UMTS, GSM, TETRA, TWT, SSP, satellite

Abstract: In this paper, we implement memoryless data predistortion in QAM trasmission over Gaussian channel. The main advantage of predistortion is the ability to reduce the back-off of an amplifier without loss of performance. Comparing three types of Solid State Amplifier, one of them distinguishes significantly better performance taking into account global degradation and bit error rate.

\section{INTRODUCTION}

The present world of telecommunication is transmitting an increasing amount of information in digital form. The number of services offered grows rapidly. Thus, higher rates of transmission and simultaneously higher quality are becoming standard requirements for different modern systems, such as pulse coded modulation for multiple voice telephony, digital audio broadcasting or digital high definition television, and $2^{\text {nd }}$ as well as $3^{\text {rd }}$ generation mobile radio systems like GSM, TETRA or UMTS. In order to reach high rates, there is, however, a second aspect related to the growing number of services that telecommunications must cope with. The 
electromagnetic frequency band, as a resource, is unique and must be used wisely, e.g., efficiently.

The modern advanced techniques like CPM, TCM or Turbo-Codes do not solve all problems which appear when the number of states in signal constellations increases. As the number of levels grows, modulation is more sensitive to propagation conditions like fading, echoes and noise as well as other linear and non-linear distortion. Among different system imperfections, there are:

- carrier frequency and phase errors due to the phase jitter of a phase-lock loop [1] at the receiver,

- errors due to a jitter of the optimum sampling instant,

- sinusoidal interference generated by a local oscillator whose frequency falls within the received signal bandwidth,

- filtering imperfections which produce linear and sinusoidal distortions depending on the amplitude and phase response of the channel filters,

- non-linear distortion.

Non-linear distortion is primarily due to the transmitter high-power amplifier (HPA) which limits considerably the bandwidth-efficiency and performance. The effect of HPA nonlinearity can be reduced by backing-off the output signal level from the amplifier's saturation point, but it reduces the transmitted signal power and, consequently, the link flat-fade margin. The link margin is a safety margin introduced in the system to improve efficiency against random disturbances. To increase this margin, the HPA should be driven as close to its saturation point as possible. Therefore, the transmitted signal power is chosen, in practice, as a trade-off between these conflicting two requirements, i.e., high amplification and low signal distortion.

The basic purpose of this work is to examine a predistortion technique for different types of HPA. Studies were done for non adaptive memoryless data predistortion. All methods are tested for 16-, 32- and 64-QAM signal constellations. The roll-off factor in the raised-cosine transmit and receive filters takes value of 0.35 .

\section{CHANNEL DESCRIPTION}

\subsection{Radio System}

The radio system operating over an AWGN channel is considered. The channel has no multipath propagation, i.e., the transmission medium only adds white Gaussian noise $\mathrm{n}(t)$ to the transmitted signal of symbols $\left\{a_{n}\right\}$. Furthermore, the timing and carrier synchronization circuits are assumed ideal, so the radio system can be represented by a simple equivalent baseband model. Omitting any predistortion at the transmitter or any 
compensation technique at the receiver, a block diagram of the radio system is sketched in Fig. 1(a), and its baseband-equivalent in Fig. 1(b), where $T$ is the symbol interval and $\delta(\cdot)$ is the Dirac delta function.

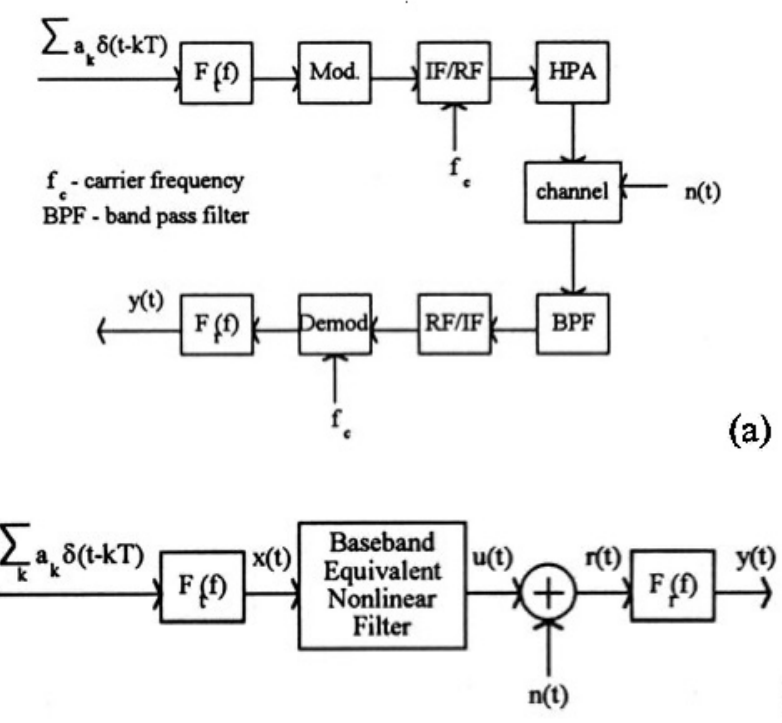

(b)

Fig. 1. (a) Block diagram of the considered system. (b) Its baseband-equivalent model.

\subsection{Radio System Modules}

\subsubsection{Filtering and Modulations}

The pulse shaping is performed at baseband and is assumed to have a raised-cosine Nyquist characteristic evenly split between the transmitter (filter $\mathrm{F}_{t}(f)$ ) and the receiver (filter $\mathrm{F}_{\mathrm{t}}(f)$ ). In the absence of HPA nonlinearity, data transmission is free of intersymbol interference ISI, and the signal-tonoise ratio SNR is maximised at the sampling instants (matched filtering). This splitting minimizes also the overlap between adjacent channels.

In Fig. 1(a), a band pass filters BPF is sketched which cancels unwanted products coming from adjacent channels. A BPF can be placed after the HPA to compensate for the spectral spreading due to the HPA itself but it introduces an additional loss of HPA power.

Successive signal states of QAM modulations are uncorrelated, and maximum-likelihood sequence estimation (MLSE) reduces to symbol-bysymbol threshold detection. 


\subsubsection{Amplifiers}

Two types of HPA's commonly used in microwave systems are travelling-wave tube (TWT) HPA's and GaAs FET HPA's (called as Solid State Power SSP). It is known that TWT HPA's present even less linear characteristics than SSP amplifiers. The HPA is assumed to have a frequency-independent memoryless bandpass nonlinearity characteristic [2]. The TWT nonlinearity is completely characterised by its AM/AM conversion

$$
a(t)=\frac{\alpha_{A} r(t)}{1+\beta_{A} r^{2}(t)}
$$

and $\mathrm{AM} / \mathrm{PM}$ conversion

$$
\psi(t)=\phi(t)+\frac{\alpha_{\mathrm{p}} r(t)}{1+\beta_{\mathrm{P}} r^{2}(t)}
$$

where $\mathrm{r}(t)$ is the magnitude and $\phi(t)$ is the phase of a point in the input constellation. $\alpha_{\mathrm{A}}, \alpha_{\mathrm{P}}, \beta_{\mathrm{A}}$ and $\beta_{\mathrm{P}}$ are coefficients identified different TWTs. Output magnitude a $(t)$ and phase $\psi(t)$ depend only on the current value of input magnitude $\mathrm{r}(t)$.

The SSP amplifier is characterized by the following equations:

$$
a(t)=\frac{v_{k} r(t)}{\left[1+\left(\frac{v_{k} r(t)}{A_{0}}\right)^{2 p_{k}}\right]^{\frac{1}{2 p_{k}}}}
$$

and

$$
\psi(t)=\phi(t)
$$

The AM/PM transition is linear while the output magnitude $a(t)$ is function of an input magnitude and some coefficients changing with the type of SSP.

To quantify the signal degradation introduced by backing-off the amplifier from its saturation point, the output back-off factor, $B \mathrm{O}_{\mathrm{o}}$, is defined by the ratio of the transmitted average signal power, $P_{\mathrm{o}}$, to the HPA's output saturation power, $P_{\text {sat, }}$ e.g.,

$$
\left.B O_{\mathrm{o}}\right|_{\mathrm{dB}}=10 \log \frac{P_{\text {sat }}}{P_{\mathrm{o}}}
$$


The study has focused on three SSP amplifiers. Two SSP amplifiers are by Sony and the third one (RF2108) is by Micro Devices. Figures $2 \div 4$ show the amplitude and phase characteristics given by producers' catalogs.
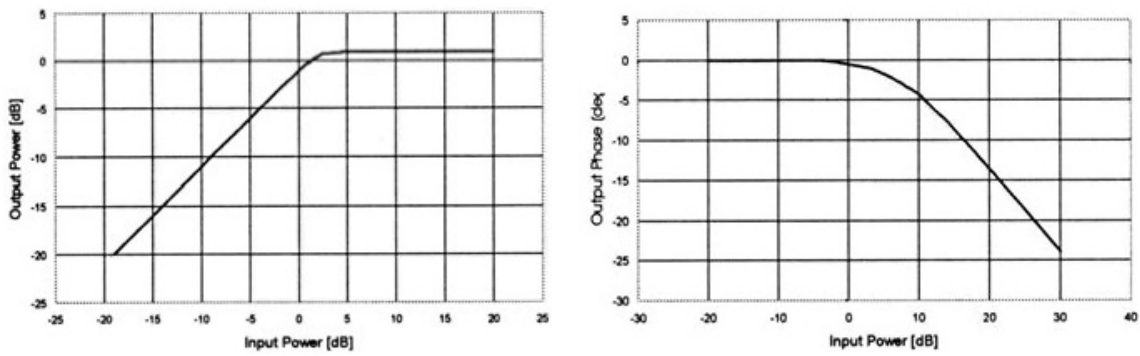

Fig. 2. Amplitude and phase characteristics of Sony's SSPI amplifier.
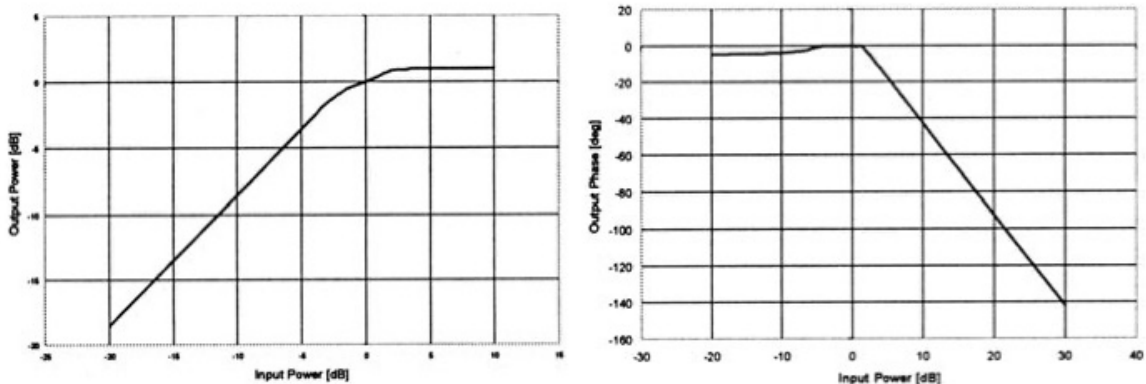

Fig. 3. Amplitude and phase characteristics of Sony's SSP2 amplifier.
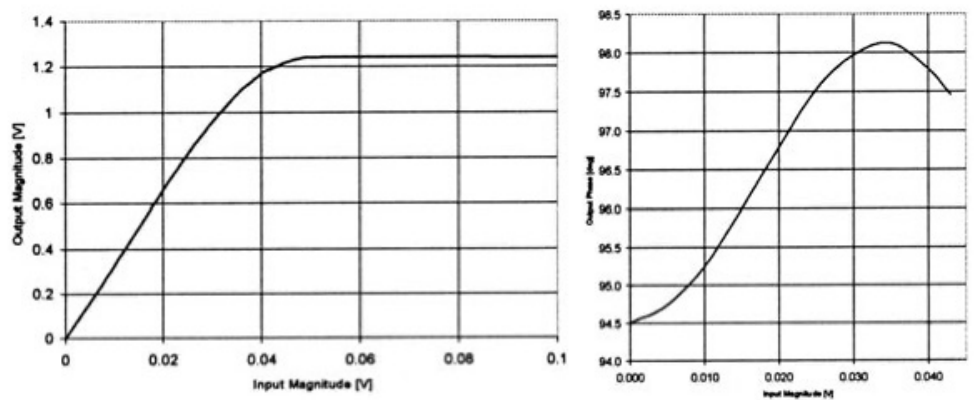

Fig. 4. Amplitude and phase characteristics of RF2108 amplifier.

\subsection{Channel Model}

Although the HPA is modelled with memoryless nonlinearity, its embedment between linear filters leads to a non-linear system with memory as a model for the overall channel. A convenient tool to represent such a system is the Volterra series [3]. 
The receiver filter output $\mathrm{y}(t)$ (as shown in Fig. 1) can be expressed as

$$
\begin{aligned}
& \mathrm{y}(t)=\sum_{k=0}^{\infty}\left[\frac{\left(\begin{array}{c}
2 k+1 \\
k
\end{array}\right)}{2^{2 k+1}}\right] \sum_{n_{1}} \sum_{n_{2}} \cdots \sum_{n_{2 k+1}} a_{n_{1}}^{*} a_{n_{2}}^{*} \cdots a_{n_{k}}^{*} a_{n_{k+1}} \cdots a_{n_{2 k+1}} . \\
& \mathrm{h}_{2 k+1}\left(t-n_{1} T, t-n_{2} T, \ldots, t-n_{2 k+1} T\right)+\mathrm{N}(t)
\end{aligned}
$$

where the terms in brackets represent the binomial coefficients, the $a_{n}$ 's are the transmitted data symbols, $T$ is the symbol interval, $\mathrm{N}(t)$ is an additive noise, and $h_{2 k+1}$ is the Volterra coefficient.

In QAM systems, the nonlinearity has two major effects. First, the clustering phenomenon reflects the linear and non-linear ISI. We obtain a cluster of points instead of a single point in the constellation. In a non-linear system, the filtering is responsible for this effect. The second effect of the nonlinearity is that the respective gravity centers of the various clusters are no longer on a rectangular grid. This distortion is called warping of the signal constellation. These two phenomena depend on the roll-off factor $\alpha$, which influence the memory of the system. As $\alpha$ decreases, the clustering becomes stronger. Moreover, the size of the clusters tends to be larger for signal points with larger amplitude. This confirms the observation from the Volterra series analysis [4] indicating that the ISI energy is concentrated in products involving the present symbol.

\section{PERFORMANCE ANALYZE}

For each modulation scheme, the carrier-to-noise ratio (CNR) degradation at the BER value of $0^{-3}$ was computed for different values of the HPA back-off.

The global degradation, defined as the sum of the HPA back-off and the CNR degradation due to non-linear distortion was then plotted as a function of the amplifier back-off. The CNR degradation is calculated in comparision with the channel with AWGN. The resulting set of convex curves gives the optimum HPA back-off for each system as well as the corresponding degradation. This measure is related to the flat-fade margin of the radio link. For an accepted degradation level one can choose a system which offers the smallest back-off.

The computation of the CNR degradation was based on generating a pseudo random data sequence, computing the corresponding discrete channel output sequence, and estimating the BER for specified CNR values. BER estimation was made using a quasi-analytic method [5], which estimates the error probability as mean of conditional probabilities that a received symbol 
without noise falls in an area determined by the treshold detector for the desired symbol. The quasi-analytic technique requires less symbols to evaluate the BER and is more precise than Monte-Carlo method.

Constellations for each modulation scheme are observed at the receiver. The same condition as for the computation of global degradation, e.g., the same back-off, must be present to compare the degradation of each constellation.

The evaluation of the BER as a function of the SNR was performed using the optimal back-off read from the global degradation curve. Additionally, the influence of HPA back-off degradation was taken into consideration, e.g., the $\mathrm{x}$-axis represents the sum of the SNR and the amplifier back-off, because the back-off can also be interpreted as SNR degradation.

\section{PREDISTORTER}

To make better use of the available HPA power, some compensation techniques can be used at the transmitter or at the receiver. Normally it is better to place compensation circuits at the transmitter because it deals with a noiseless signal. The use of fixed or adaptive analog signal predistortion at IF or RF stage of the transmitter has almost become common practice in digital microwave radio systems. Adaptive predistortion has the advantage of automatically coping with any time-variations of the HPA response due to temperature variations and aging, while the intervention of a human operator is occasionally necessary in the case of fixed predistortion. Furthermore, predistortion has to be adaptive if it needs to be incorporated in a transmitter with automatic control of the transmitted power like in base stations and in mobile units of almost all mobile systems. Another approach to handle the non-linear distortion problem is the data predistortion which consists of modifying the input signal constellation so as to obtain the desired constellation after the HPA nonlinearity.

\subsection{TWT predistorter}

The key idea is that if the HPA nonlinearity can be characterized by a pair of functions as given by (1) and (2), the desired signal constellation can be obtained at the (demodulated) HPA output by driving the modulator by an appropriately predistorted constellation.

By computing a pair of (predistorted) coordinates for each point of the desired constellation, the effect of HPA nonlinearity can be eliminated completely. This is true when all pulse shaping is performed after HPA, but in this case, where some filtering precedes the HPA, data predistortion can only compensate for constellation warping. It does not reduce (and experience has shown that it can even enhance) the clustering of the signal 
constellation which reflects non-linear ISI. The baseband block diagram of this technique is depicted in Fig. 5.

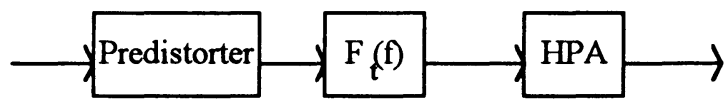

Fig. 5. Baseband block diagram of memoryless data predistortion.

The inverse of the TWT equations (1) and (2) leads to the following ones

$$
\mathrm{r}(t)=\frac{\alpha_{\mathrm{A}}}{2 \mathrm{c}(t) \beta_{\mathrm{A}}}\left[1-\sqrt{1-\left(2 \mathrm{c}(t) \sqrt{\beta_{A}}\right)^{2}}\right] \text { if } \mathrm{c}(t)<\frac{\alpha_{\mathrm{A}}}{2 \sqrt{\beta_{\mathrm{A}}}}
$$

and

$$
\phi(t)=\varphi(t)-\frac{\alpha_{\mathrm{p}} \mathrm{c}^{2}(t)}{1+\beta_{\mathrm{p}} \mathrm{c}^{2}(t)} \text { if } \mathrm{c}(t)<\frac{\alpha_{\mathrm{A}}}{2 \sqrt{\beta_{\mathrm{A}}}}
$$

\subsection{SSP predistorter}

The predistorter characteristic is an inverse function of the SSP amplifier equations (3) and (4).

$$
\begin{aligned}
& r(t)=\frac{1}{v_{k}} \frac{c(t)}{\left[1-\left(\frac{c(t)}{A_{0}}\right)^{2 p_{k}}\right]^{\frac{1}{2 p_{k}}}} \text { when } \mathrm{c}(t)<\mathrm{A}_{0} \\
& \phi(t)=\varphi(t)
\end{aligned}
$$

The Tab. 1 contains the values of parameters of the predistorter obtained with least-square fitting of equation (3) to real characteristic (figures $2 \div 4$ ) of SSP amplifiers.

Table 1. Parameters of predistorters for SSPs.

\begin{tabular}{|l|l|l|l|}
\hline Amplifier & $v_{k}$ & $A_{0}$ & $p_{k}$ \\
\hline SSP1 & 1.65 & 1 & 3.4 \\
\hline SSP2 & 1.82 & 1.04 & 1.65 \\
\hline RF2108 & 1.3 & 1 & 5 \\
\hline
\end{tabular}

For simulation purposes, all characteristics of amplifiers and predistorters were normalized in order that the saturation point has the coordination of $(1,1)$. 


\section{SIMULATION RESULTS}

Using QAM signal formats, we made computer simulations to compare SSP amplifiers. In what follows, we give the main results of a simulation study which aimes at comparing predistortion technique on Gaussian channel. The simulations were carried out using a QAM system with ZF equalisation.

Figures 6 and 7 show global degradation for 16- and 64-QAM and three types of discussed amplifiers. The performance of an applied predistorter is presented on figures 8 and 9.

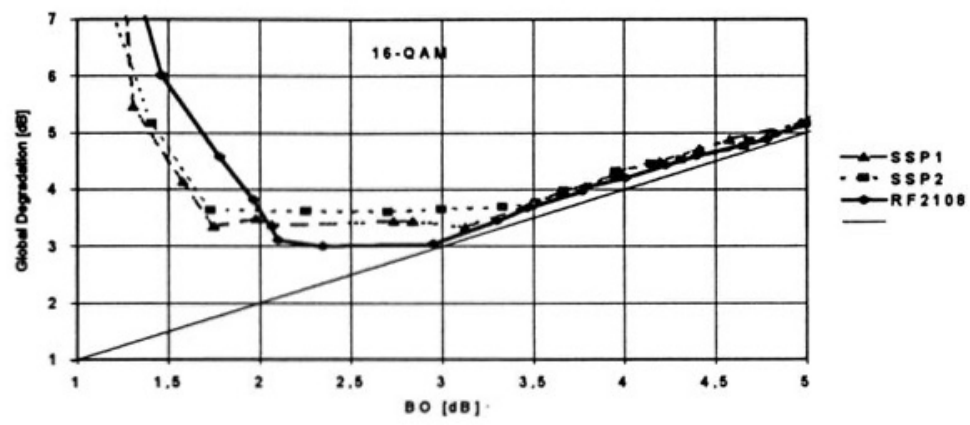

Fig. 6. Global degradation for 64-QAM modulation scheme and three types of amplifiers. Predistorter is on

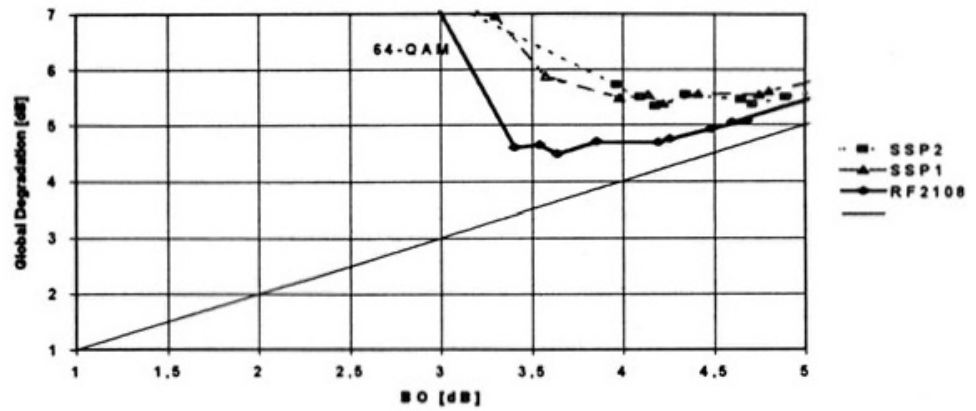

Fig. 7. Global degradation for 64-QAM modulation scheme and three types of amplifiers. Predistorter is on. 


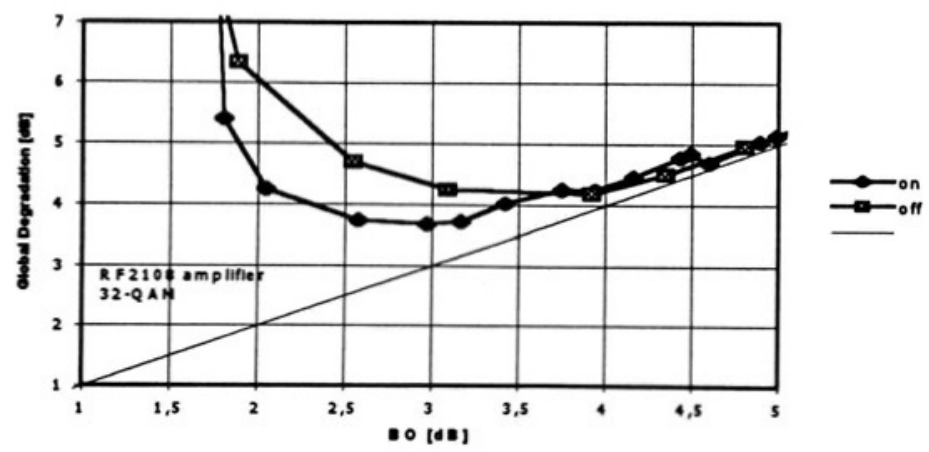

Fig. 8. Comparison of global degradation when predistorter is on and off for 32-QAM.

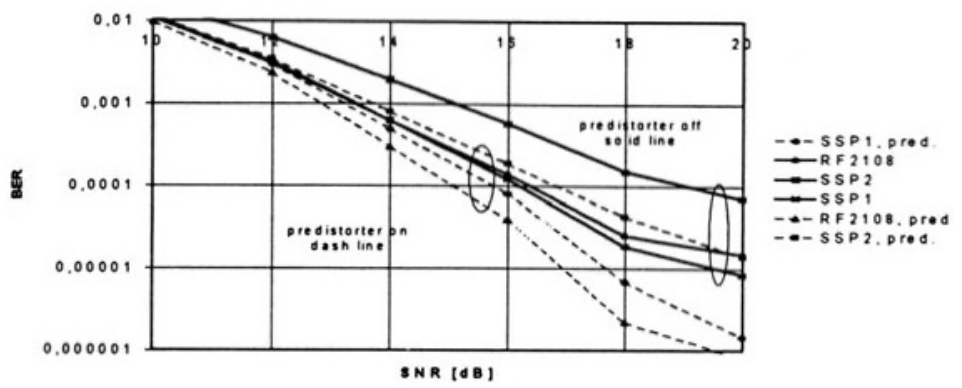

Fig. 9. Comparison of BER when predistorter is on and off for 32-QAM.

\section{CONCLUSIONS}

The memoryless data predistortion is easy to implement and offers some gain compared to a system without predistortion but it cannot compensate for the memory introduced by the filters situated before and after the HPA.

Using that predistorter, the global degradation can be reduced by up-to $1 \mathrm{~dB}$ and back-off may be less of about $1.5 \mathrm{~dB}$. This yields better BER performance. For BER equal $10^{-4}$ using 32-QAM modulation scheme one can gain about $2.5 \mathrm{~dB}$ for SSP2, $1.3 \mathrm{~dB}$ for RF2108 and only $0.2 \mathrm{~dB}$ for SSP1 amplifier.

Among considered Solid State Amplifiers, one of Micro Devices offers the best performance, e.g., less back-off and less degradation than the amplifiers by Sony.

That modern technique is very useful to help us achieve the goal of transmitting the largest amount of information possible on the available bandwidth. The new bandwidth efficient techniques can be used even more 
efficiently on systems such as mobile radio, or other hertzien type channels for which the price of the bandwidth and number of users keeps increasing.

\section{REFERENCES}

[1] J. G. Proakis, "Digital Communications," New York: McGraw-Hill, 1983.

[2] G. Karam and H. Sari, "Generalized Data Predistortion Using Intersymbol Interpolation," Philips Journal of Research, vol. 46, no. 1, pp. 1-22, 1991.

[3] R. D. Gitlin, J. F. Hayes and S. B. Weinstein, "Data Communications Principles," New York: Plenul Press, 1992.

[4] G. Karam, "Analyse et compensation des distortions non linéaires dans les faisceaux hertziens numerique", Télécom Paris (ENST), octobre 1989.

[5] M. J. Jeruchim, "Techniques for Estimating the Bit Error Rate in the Simulation of Digital Communication Systems," IEEE JSAC, vol. SAC-2, pp. 153-170, January 1984. 